\title{
Effects of metformin on the expression of AMPK and STAT3 in the spinal dorsal horn of rats with neuropathic pain
}

\author{
ANQI GE ${ }^{1 *}$, SHU WANG $^{2 *}$, BEI MIAO ${ }^{3,4}$ and MING YAN ${ }^{5}$ \\ ${ }^{1}$ Jiangsu Province Key Laboratory of Anesthesiology, Clinic Skill Center, Xuzhou Medical University, \\ Xuzhou, Jiangsu 221000; ${ }^{2}$ Department of Anesthesiology, The Third People's Hospital, Yancheng, Jiangsu 224001; \\ ${ }^{3}$ Laboratory of Gastroenterology, Xuzhou Medical University, Xuzhou, Jiangsu 221000; ${ }^{4}$ Department of Gastroenterology; \\ ${ }^{5}$ Jiangsu Province Key Laboratory of Anesthesiology, Department of Anesthesiology, The Affiliated \\ Hospital of Xuzhou Medical University, Xuzhou, Jiangsu 221000, P.R. China
}

Received February 10, 2017; Accepted December 29, 2017

DOI: $10.3892 / \mathrm{mmr} .2018 .8541$

\begin{abstract}
Neuropathic pain (NP) is a frustrating and burdensome problem. Current treatments for NP have unendurable side effects and/or questionable efficacy, and once these therapies are stopped, the symptoms often return. Thus, novel drugs are needed to enhance the effectiveness of treatments for NP. One novel target for pain treatments is adenosine monophosphate-activated protein kinase (AMPK), which regulates a variety of cellular processes, including protein translation, which is considered to be affected in NP. Metformin is a widely available drug that possesses the ability to activate AMPK. The signal transducer and activator of transcription 3 (STAT3) pathway plays an important role in neuroinflammation. The present study investigated the analgesic effect of metformin on NP induced by chronic constriction injury (CCI), and the influence of metformin on the expression of AMPK and STAT3 in the spinal dorsal horn (SDH). In CCI rats, paw withdrawal latencies in response to thermal hyperalgesia were significantly shorter, while phosphorylated (p)-AMPK was expressed at lower levels and p-STAT3 was expressed at higher levels in the SDH. Administering intraperitoneal injections
\end{abstract}

Correspondence to: Professor Bei Miao, Laboratory of Gastroenterology, Xuzhou Medical University, 99 West Huaihai Road, Quanshan, Xuzhou, Jiangsu 221000, P.R. China

E-mail: benjaminmb@163.com

Professor Ming Yan, Jiangsu Province Key Laboratory of Anesthesiology, Department of Anesthesiology, The Affiliated Hospital of Xuzhou Medical University, 99 West Huaihai Road, Quanshan, Xuzhou, Jiangsu 221000, P.R. China

E-mail: yjy3001@163.com

*Contributed equally

Key words: adenosine monophosphate-activated protein kinase, signal transducer and activator of transcription 3, metformin, neuropathic pain, spinal dorsal horn, glia of metformin $(200 \mathrm{mg} / \mathrm{kg})$ for 6 successive days activated AMPK and suppressed the expression of p-STAT3, in addition to reversing hyperalgesia. Finally, metformin inhibited the activation of microglia and astrocytes in the SDH, which may explain how it alleviates NP.

\section{Introduction}

The neuropathic pain (NP) was newly defined as pain induced by a disease or lesion of the somatosensory system by International Association for the Study of Pain (IASP) (1). Due to the unsatisfactory therapeutic regimens for NP, a multitude of affected patients cannot alleviate their pain (2). Hence, understanding of pathogenesis underlying NP and quest for novel effective drugs for the therapeutics of NP is essential.

Chronic constriction injury (CCI) models have both peripheral nerve injury and inflammatory components which can mimic NP in humans and are effective for investing the mechanisms underlying NP $(3,4)$.

Herein, the effect of a safe and widely used oral hypoglycemic drug, metformin, was invested on NP. Metformin may restrain peripheral nerve injury-induced NP (5), and it also may potentially decrease lumbar radiculopathy pain in humans (6). However, the mechanism of the inhibitive effects of metformin on NP has not been investigated in vivo in previous studies. We innovatively explored the effects of metformin on the spinal cords of rats in a CCI-induced NP model.

Adenosine monophosphate-activated protein kinase (AMPK) is a serine/threonine protein kinase that is central to cellular metabolic processes and energy balance maintenance, inflammation, cancer, neurodegenerative diseases (7-9). Studies have also shown that AMPK plays a key role in pain $(10,11)$. Moreover, metformin is validated to be an indirect activator of AMPK $(12,13)$. Therefore, we speculated that AMPK might be involved in the analgesic effects of metformin.

Metformin reportedly inhibits signal transducer and activator of transcription 3 (STAT3) in tumor cells $(14,15)$. In addition, the STAT3 pathway is activated in the spinal dorsal horn (SDH) in both NP (16-18) and spinal cord injury models (19). However, whether the STAT3 pathway is involved 
in the analgesic effe-cts of metformin on NP remained unclear. Here, we evaluated the analgesic effects of metformin and its potential effects on the STAT3 signaling pathway in the SDH in rodent models of NP.

The activation of spinal glial cells plays an crucial role in NP $(20,21)$. Further, AMPK and STAT3 are implicated in the regulation of the activation of glial cells $(22,23)$. We also investigated the effect of metformin on microglia and astrocytes in the CCI models. Collectively, our data reveal a potential mechanism underlying the analgesic effect of metformin and may shed new light on the therapeutics for NP.

\section{Materials and methods}

Animals. Male Sprague-Dawley rats (weighing 200-230 g, aged 6-8 weeks) were obtained from the experimental animal center of Xuzhou Medical University (Xuzhou, China). The animals were housed under the following controlled conditions: A $12 \mathrm{~h}$ light-dark cycle (lights on from 6 a.m. to 6 p.m.) at a steady temperature of $25^{\circ} \mathrm{C} \pm 1^{\circ} \mathrm{C}$ with free access to water and food. All protocols were approved by the Institutional Animal Care and Use Committee at Xuzhou Medical University. The experimental procedures were conducted according to the ethical guidelines for the care and use of laboratory animals of the National Institutes of Health (NIH Publications, no. 8023, revised 1978) and the IASP. Effort was made to decrease the number of animals used and to reduce animal suffering.

CCI of the sciatic nerve. The CCI rat model was established complied to a previously described method (24). The rats were anesthetized using $10 \%$ chloral hydrate $(0.3 \mathrm{ml} / 100 \mathrm{~g}$, i.p.). The left sciatic nerve was exposed using a small incision at the mid-thigh level. A constriction injury was induced proximal to the trifurcation of the sciatic nerve by making loose ligations with four 4-0 silk threads (1 mm apart). In the sham-operated group, the nerve was exposed without ligations. The wound was then treated using antibiotics (penicillin) and closed layer-by-layer.

Experimental groups. A total of 112 rats were used across all experiments. Behavioral assessment and fasting blood glucose test consists of two parts. Part I: The rats were randomly divided into the 4 groups $(n=8)$ : Sham + normal saline (sham + NS) group, sham + metformin group, CCI + NS group, and CCI + metformin group. The rats received i.p. injections of $5 \%$ metformin in NS $(200 \mathrm{mg} / \mathrm{kg}$, Sigma, USA) or an equal dose of NS once per day for 6 consecutive days beginning on the 5th day after surgery. The dose of metformin was selected according to a previous reference (5). Behavioral assessment was performed on days-1 (the day before surgery), and 3, 5, 7, 10, 14 after surgery. The data of-1th day were detected as baseline. On the 5th day after surgery, the behavioral assessment was performed before metformin and saline administered, whereas on the 7th and 10th days, the behavioral assessment was performed two $h$ after the injections. Fasting blood glucose levels were measured after the behavioral assessment on days $-1,3,5,7,10,14$. The blood was taken from the tail vein. Part II: The rats were randomly divided into 5 groups $(n=8)$ : $\mathrm{CCI}$ group, $\mathrm{CCI}+\mathrm{NS}$ group, $\mathrm{CCI}+$ metformin group, $\mathrm{CCI}+$ compound $\mathrm{C}$ (AMPK inhibitor) group, and CCI + metformin + compound $\mathrm{C}$ group. The rats received intraperitoneal injections of metformin or an equal dose of NS from 5th day to 10th day according to the same method mentioned above. Compound C (30 ug) was intrathecal administration on 10th day in $\mathrm{CCI}+$ compound $\mathrm{C}$ group and $\mathrm{CCI}+$ metformin + compound $\mathrm{C}$ group. Behavioral assessment was performed on days $-1,3,5,7,10,14$ after surgery.

Western blot analysis and immunofluorescence staining. The rats were randomly divided into sham $+\mathrm{NS}$, sham + metformin, $\mathrm{CCI}+\mathrm{NS}$, and CCI + metformin groups $(\mathrm{n}=10)$. In each group, 4 rats were used for western blot and 6 rats for immunofluorescence staining. On the 10th day, the rats were deeply anesthetized, then sacrificed, and the L4-L6 spinal cord segments were collected for either immunofluorescence staining or western blot analysis.

Reagents. The major reagents which were employed in this study were metformin (Sigma-Aldrich, USA), compound C (Sigma-Aldrich, USA), rabbit polyclonal anti-phosphorylated (p)-AMPK (Cell Signaling Technology; Bioworld Technology Inc., St. Louis Park, MN, USA), rabbit polyclonal anti-p-STAT3 (Cell Signaling Technology; Bioworld Technology Inc.); rabbit polyclonal anti-AMPK, rabbit polyclonal anti-STAT3, rabbit polyclonal anti-GAPDH (all Bioworld Technology Inc.), goat polyclonal anti-ionized calcium binding adaptor molecule 1 (Iba-1; Abcam, Cambridge, UK), mouse polyclonal anti-glial fiber acidic protein (GFAP; Cell Signaling Technology, Inc., Danvers, MA, USA), Alexa 488 donkey anti-rabbit IgG, Alexa 546 donkey anti-goat IgG, Alexa 594 donkey anti-mouse IgG (all Invitrogen, Carlsbad, CA, USA), and HRP-conjugated goat anti-rabbit secondary antibodies (Beyotime Institute of Biotechnology, Shanghai, China).

Behavioral assessment. Thermal hyperalgesia was evaluated by measuring paw withdrawal latency (PWL) in response to heat stimulation. A plantar heat analgesia meter model 390 (IITC Life Science Inc., Woodland Hills, CA, USA) was used according to the protocols described by Hargreaves et al to provide a heat source (25). Each rat was placed in a transparent acrylic chamber that was placed on a glass plate. Following a one-hour adaptation period, the middle of the plantar surface of the left hind paw was exposed to a radiant heat source using a beam of light. The length of time between the start of the light beam and the foot lift was recorded as the PWL. To avoid damaging the tissue, an automatic $25 \mathrm{sec}$ cutoff was set. This thermal stimulus was repeated five times for each paw at an interval of $5 \mathrm{~min}$. The test was conducted in a noise-free and temperature-controlled $\left(25-26^{\circ} \mathrm{C}\right)$ room. The intensity of the thermal stimulation was maintained at the same level throughout the entire study.

Intrathecal injection procedure. Rats were anesthetized with inhalation of isoflurane and placed in a prone position on the operating table. Midpoint between the tips of the iliac crest was labeled as the fifth to sixth lumbar intervertebral space (L5-L6). Intrathecal injection was performed by a micro 

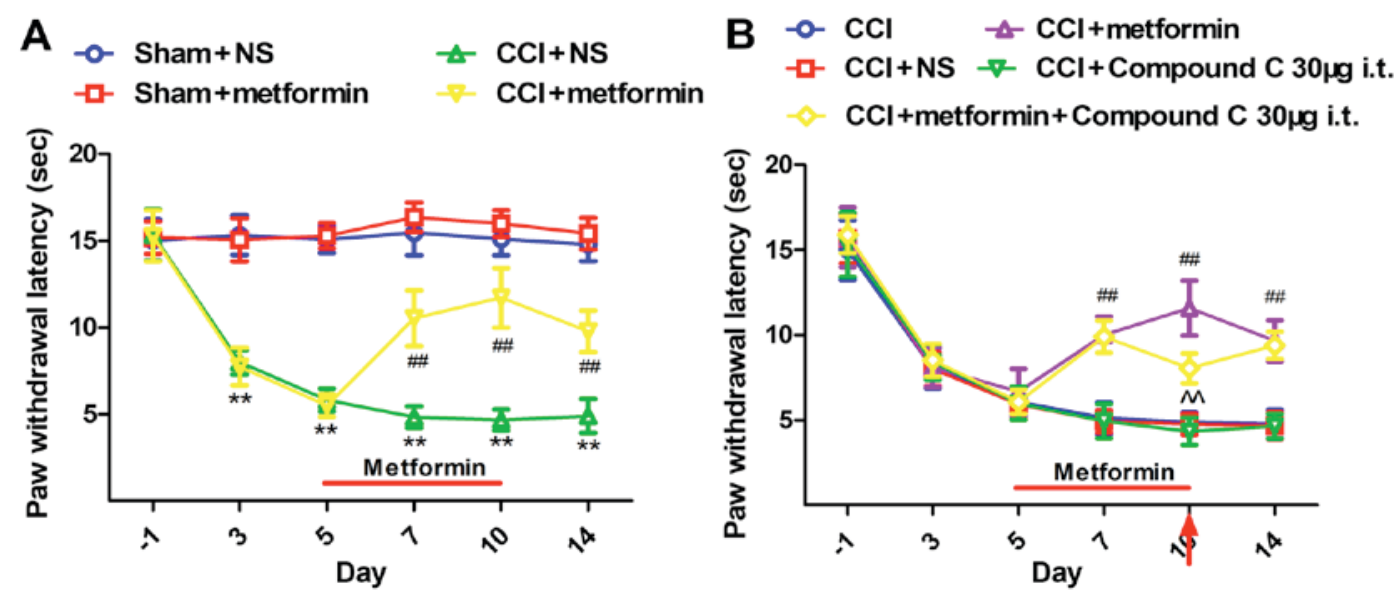

Figure 1. Intraperitoneal administration of metformin attenuates neuropathic pain in CCI rats. (A) CCI rats exhibited a significant decrease in the PWLs 3-14 days post-surgery. The intraperitoneal administration of metformin $(200 \mathrm{mg} / \mathrm{kg}$, once per day on days $5-10)$ attenuated thermal hyperalgesia $(\mathrm{n}=8$; ${ }^{* *} \mathrm{P}<0.01$ vs. sham $+\mathrm{NS} ;{ }^{* \#} \mathrm{P}<0.01$ vs. $\left.\mathrm{CCI}+\mathrm{NS}\right)$. (B) The intrathecal administration of compound $\mathrm{C}(30 \mu \mathrm{g})$ on day 10 could reverse the analgesic effect of metformin ( $\mathrm{n}=8 ;{ }^{\# \#} \mathrm{P}<0.01$ vs. $\mathrm{CCI}+\mathrm{NS},{ }^{\wedge} \mathrm{P}<0.01$ vs. $\mathrm{CCI}+$ metformin). NS, normal saline; PWL, paw withdrawal latency; CCI, chronic constriction injury.

syringe, which was inserted into the subarachnoid space of the spinal cord between the L5 and L6 spinous processes. Intrathecal injection was confirmed by observation of an obvious tail flick.

Western blot analysis. The rats were anesthetized with $10 \%$ chloral hydrate $(0.3 \mathrm{ml} / 100 \mathrm{~g}$, i.p. $)$ and killed by decapitation. The dissected L4-L6 spinal dorsal horn samples were rapidly isolated and frozen in liquid nitrogen and thereafter placed in long-term storage at $-80^{\circ} \mathrm{C}$ till used. The tissue samples were homogenized in RIPA buffer containing protease inhibitors and phosphatase inhibitors and centrifuged for $15 \mathrm{~min}\left(12,000 \mathrm{~g}\right.$, at $\left.4^{\circ} \mathrm{C}\right)$, with the supernatant collected. The protein concentration of the supernatant was determined by a Bicinchoninic Acid Protein Assay kit (Beyotime Institute of Biotechnology) according to the protocol described in the previous study (26). Equal amount of protein samples were separated by $10 \%$ sodium dodecyl sulfate-polyacrylamide gel electrophoresis and transferred to polyvinylidene difluoride membranes (EMD Millipore, Billerica, MA, USA). After thrice rinsed, the membranes were blocked in $3 \%$ bovine serum albumin for $2 \mathrm{~h}$ at room temperature $(\mathrm{r} / \mathrm{t})$ and incubated with the following primary antibodies: Rabbit anti-AMPK (1:300; Bioworld Technology Inc.), rabbit anti-p-AMPK (1:600; Cell Signaling Technology), rabbit anti-STAT3 (1:500; Bioworld Technology Inc.), rabbit anti-p-STAT3 (1:300, Cell Signaling Technology), and rabbit anti-GAPDH (1:2,000; Bioworld Technology Inc.) for overnight at $4^{\circ} \mathrm{C}$. After three washes, the membranes were incubated with HRP-conjugated goat anti-rabbit secondary antibodies for $1 \mathrm{~h}$ (1:1,000; Beyotime Institute of Biotechnology) at $\mathrm{r} / \mathrm{t}$. The bands were visualized with the use of ECL and X-film. The densities of the protein band were quantified using Image J software (NIH, Bethesda, MD, USA).

Immunofluorescence histochemistry. The rats were deeply anesthetized using $10 \%$ chloral hydrate $(0.3 \mathrm{ml} / 100 \mathrm{~g}$, i.p. $)$ and intracardially perfused with $300 \mathrm{ml}$ of NS followed by $300 \mathrm{ml}$ of $4 \%$ paraformaldehyde (PFA) in phosphate buffer (PB; 0.1 M; pH 7.2-7.4; $\left.4^{\circ} \mathrm{C}\right)$. The spinal cords were quickly isolated and post-fixed in $4 \%$ PFA for $2 \mathrm{~h}$ at $4{ }^{\circ} \mathrm{C}$. Subsequently, the L4-L6 spinal cord samples were equilibrated in a $30 \%$ sucrose solution at $4^{\circ} \mathrm{C}$ until the sample blocks dropped to the bottom. The tissues were embedded in tissue freezing medium and sliced at thickness of $35 \mu \mathrm{m}$ by a cryostat (Leica CM1800; Heidelberg, Germany). After washing thrice with $1 \mathrm{X}$ PBS, selected sections were blocked in $1 \mathrm{X}$ PBS containing 3\% Triton-X-100 (PBST) and 10\% donkey serum for $2 \mathrm{~h}$ at $\mathrm{r} / \mathrm{t}$ and incubated with the following primary antibodies in PBST: rabbit anti-p-AMPK (1:200; Bioworld Technology Inc.), rabbit anti-p-STAT3 (1:50; Bioworld Technology Inc.), mouse anti-GFAP (1:400; Cell Signaling Technology), or goat anti-Iba-1 (1:300; Abcam) for $48 \mathrm{~h}$ at $4^{\circ} \mathrm{C}$. The slices were then washed 3 times ( 5 min each) with PBS and incubated with Alexa 488 donkey anti-rabbit IgG, Alexa 546 donkey anti-goat IgG or Alexa 594 donkey anti-mouse IgG (all 1:200; Invitrogen) for $2 \mathrm{~h}$ at $\mathrm{r} / \mathrm{t}$. Images were captured using a confocal laser microscope (FV1000; Olympus, Corp., Tokyo, Japan) and analyzed by Image Pro-Plus 6.0 software (Media Cybernetics, Silver Spring, MD, USA). Five slices for each spinal cord sample were randomly selected for statistical analysis.

Statistical analyses. The data were analyzed using GraphPad Prism 5.0 Software (GraphPad Software, La Jolla, CA, United States). All data are shown as the mean \pm SD. The normal distribution and variance homogeneity were tested. One-way ANOVA was used to analyze the western blot and immunofluorescence results across the four groups. Two-way ANOVA was used for the PWLs and fasting blood glucose values, which included two factors of time and group. If a significant difference was found, a post hoc Bonferroni multiple test was used to detect differences within the groups. $\mathrm{P}<0.05$ was considered to indicate a statistically significant difference.

\section{Results}

Behavioral outcomes and blood glucose values. To investigate the influence of metformin on CCI rats, we measured 
PWLs on days $1,3,5,7,10$, and 14 after surgery (Fig. 1). There were no differences in the PWLs between the sham + NS and sham + metformin groups. On days 3-14, the PWLs in the rats in the CCI + NS group were significantly lower than those in the sham + NS group. Meanwhile, the PWLs of the CCI rats that were injected with metformin (200 mg/kg, once a day in days 5-10) were markedly longer than those in the CCI + NS group $(\mathrm{P}<0.01)$, but shorter than those in the sham + NS and sham + metformin groups. Even after the therapy was stopped for 4 days on day 14, the PWLs in the CCI + metformin group were still considerably higher than those in the CCI + NS group $(\mathrm{P}<0.01)$ (Fig. 1A). These data revealed that the analgesic ability of metformin was sustained.

Fasting blood glucose values were collected after the same fasting time in the 4 groups following the behavioral assessment on days 1, 3, 5, 7, 10 and 14 post-surgery. There was no difference in blood glucose values among the four groups. metformin did not affect blood sugar values in the sham-operated rats and CCI rats (Fig. 2).

We further studied the relationship of metformin and the NP by AMPK inhibitor (compound C). On the 10th day, the PWLs in the rats in the CCI + metformin + compound $\mathrm{C}$ group were significantly lower than those in the $\mathrm{CCI}+$ metformin group $(\mathrm{P}<0.01)$ (Fig. 1B). We demonstrated that compound $\mathrm{C}$ could reverse the analgesic effect of metformin.

Activation and distribution of AMPK in spinal cords after CCI and metformin administration. To further explore how metformin alleviates NP in CCI rats, we determined p-AMPK and AMPK levels in the spinal dorsal horns of rats on day 10 after the final metformin or NS treatment administered. The results of western blot analysis showed that the expression level of p-AMPK was lower in the CCI + NS group than that in the sham $+\mathrm{NS}$ group $(\mathrm{P}<0.01)$ and the expression level of $\mathrm{p}$-AMPK was significantly higher in the $\mathrm{CCI}+$ metformin group than that in the CCI + NS group $(\mathrm{P}<0.01)$. However, the expression level of AMPK in the SDH was not significantly different among the four groups of rats (Fig. 3).

Next, we investigated the distribution of p-AMPK in the SDH by immunofluorescent staining. We found that p-AMPK was widely distributed in the SDH but mainly concentrated in lamina I and II. The mean fluorescence intensity and the number of cells that were positively stained for $\mathrm{p}$-AMPK were significantly lower in the CCI + NS group than those in the sham + NS group $(\mathrm{P}<0.01)$ and significantly higher in the $\mathrm{CCI}+$ metformin group than those in the $\mathrm{CCI}+\mathrm{NS}$ group $(\mathrm{P}<0.01)$ (Fig. 4).

These data showed that $\mathrm{p}$-AMPK was widely distributed in the SDH of rats. In the NP model of CCI rats, the expression of p-AMPK was suppressed, and metformin activated AMPK in the SDH via phosphorylation, thereby inhibiting the development of NP.

Activation and distribution of STAT3 in SDH after CCI and metformin administration. We further investigated whether metformin inhibited the activation of STAT3 in the spinal cords of CCI rats by exploring the expression of p-STAT3 and STAT3 in the dorsal horn. The expression level of p-STAT3

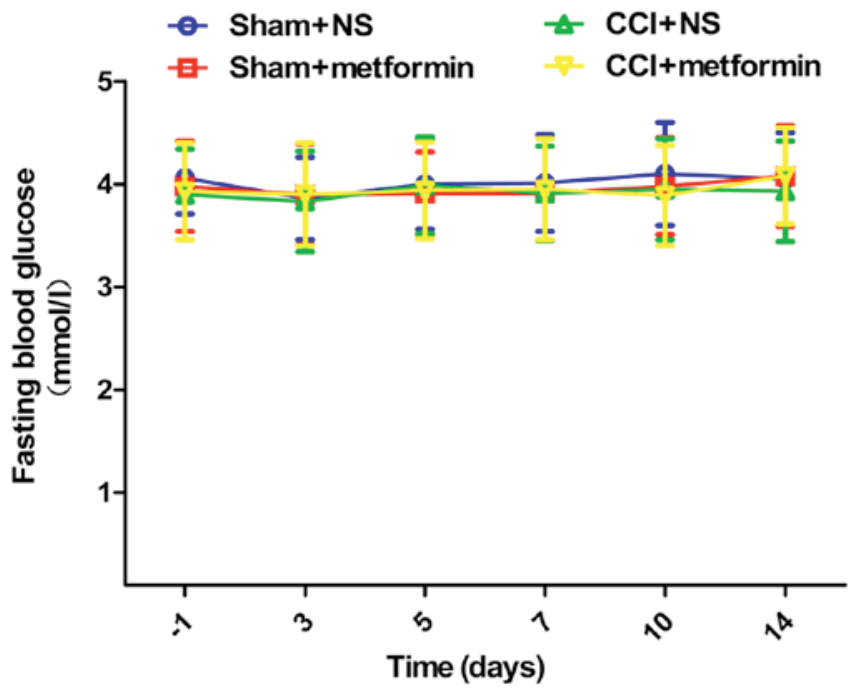

Figure 2. Fasting blood glucose levels in the sham + NS, sham + metformin, $\mathrm{CCI}+\mathrm{NS}$ and CCI + metformin groups. Metformin had no effect on fasting blood glucose levels in rats in the 4 groups $(n=8 ; P>0.05)$. NS, normal saline; CCI, chronic constriction injury.

in the spinal cord was significantly increased in the CCI + NS group than that in the sham + NS group $(\mathrm{P}<0.01)$, and the expression level of p-STAT3 was significantly decresed in the $\mathrm{CCI}+$ metformin group than that in the $\mathrm{CCI}+\mathrm{NS}$ group $(\mathrm{P}<0.01)$, with no apparent difference in the level of STAT3 in four groups of rats (Fig. 5).

The results of immunofluorescence histochemistry showed that p-STAT3 was widely distributed, mainly in laminas I and II of the SDH. The p-STAT3 expression was rarely detected in the sham + NS rats. However, the number of positively stained cells and the mean fluorescence intensity for p-STAT3 in the dorsal horn were higher in the CCI + NS rats than those in the sham $+\mathrm{NS}$ rats $(\mathrm{P}<0.01)$. In addition, the values were lower in the $\mathrm{CCI}+$ metformin group than those in the CCI + NS group $(\mathrm{P}<0.01)$ (Fig. 6).

Our results in this NP model indicated that the STAT3 signaling pathway was activated which demonstrated by the increased expression of p-STAT3, and this might be one of the causes of NP. Metformin prevented spinal dorsal horn STAT3 phosphorylation, which might account for its ability to alleviate NP.

Metformin injection suppressed the activation of microglia and astrocytes in the SDH. Glial cell activation plays a key role in the occurrence and maintenance of pain (27). In this study, we further explored whether metformin affects microglia and astrocytes. Immunofluorescence staining was performed to detect the expression of the microglia marker Iba-1 and the astrocyte marker GFAP. Consistent with previous findings, our results showed that Iba-1 and GFAP are widely distributed in the SDH. Compared with the sham + NS group, the mean fluorescence intensity for Iba-1 was higher and the number of positively stained cells for Iba-1 increased in the SDH in the CCI + NS group $(\mathrm{P}<0.01)$ (Fig. 7A). Similar findings have been found in astrocytes (Fig. 7B). Moreover, microglia and astrocytes were morphologically activated after CCI. And Compared with the CCI + NS group, the activation 
A p-AMPK GAPDH

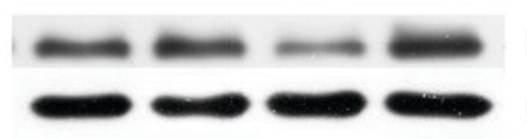

$62 \mathrm{kDa}$

$36 \mathrm{kDa}$

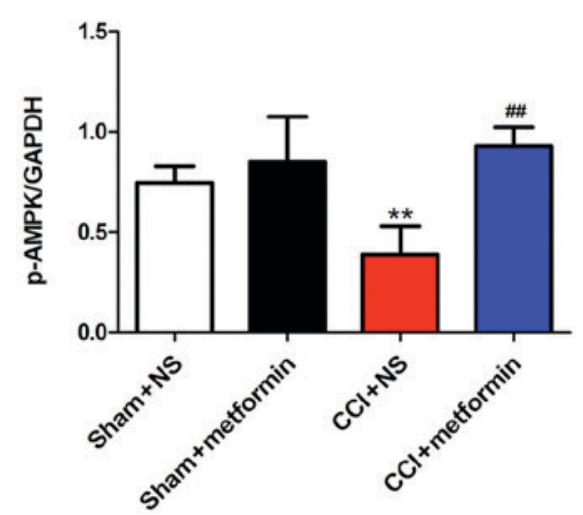

B
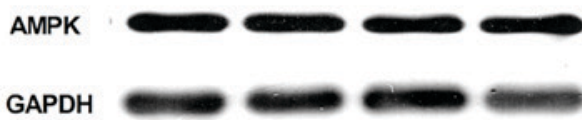

$62 \mathrm{kDa}$

GAPDH

$36 \mathrm{kDa}$

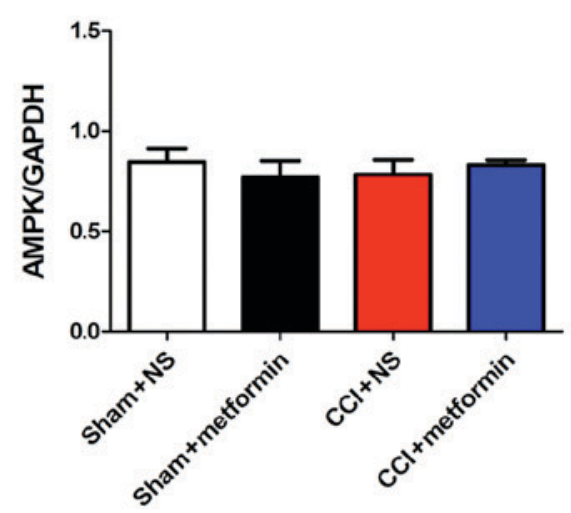

Figure 3. Metformin activated AMPK in the spinal cords of CCI rats. (A) Metformin increased AMPK phosphorylation levels in the spinal cord after CCI ( $\mathrm{n}=4$; ${ }^{* *} \mathrm{P}<0.01$ vs. sham $+\mathrm{NS} ;{ }^{\# \#} \mathrm{P}<0.01$ vs. CCI $+\mathrm{NS}$ ). (B) There was no significant difference in the expression of AMPK among the four groups $(\mathrm{n}=4 ; \mathrm{P}>0.05)$. Tissues were collected on day 10 after CCI. AMPK, adenosine monophosphate-activated protein kinase; NS, normal saline; CCI, chronic constriction injury.

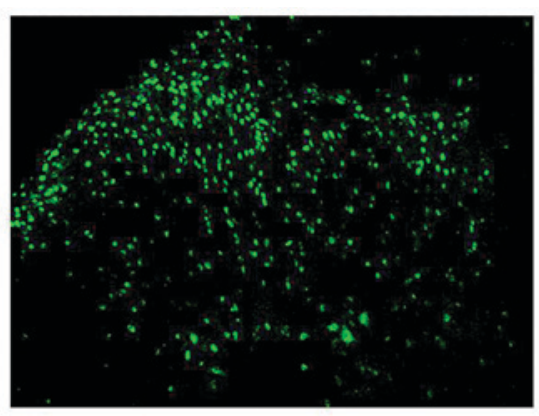

Sham + NS

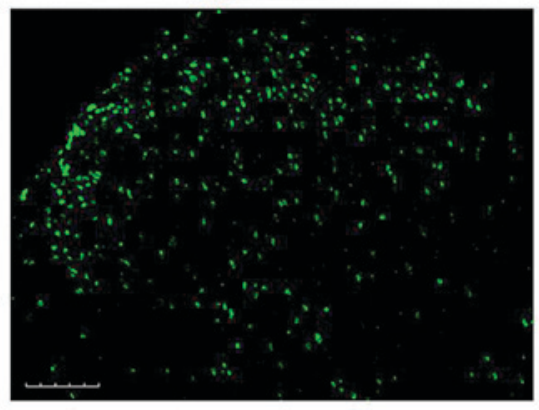

$\mathrm{CCl}+\mathrm{NS}$

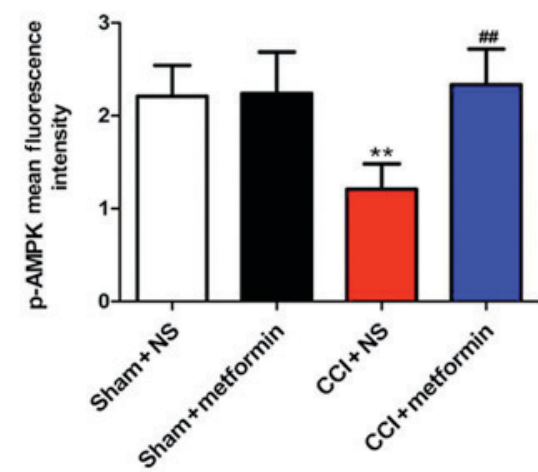

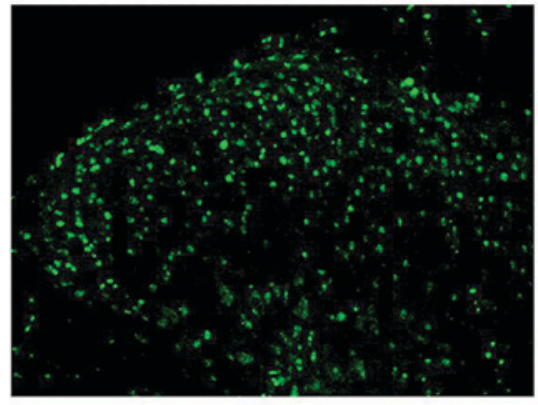

Sham+metformin

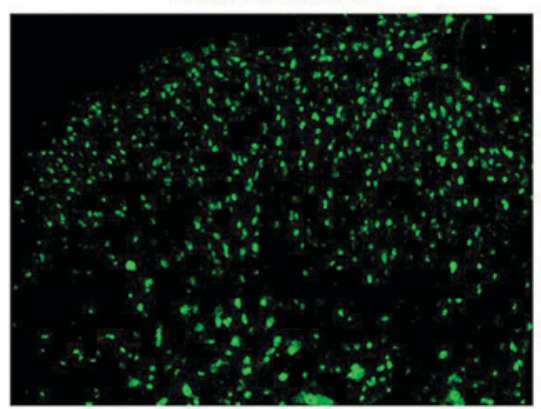

$\mathrm{CCl}+$ metformin

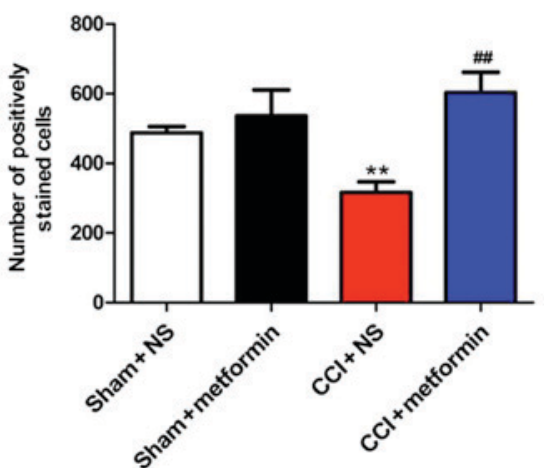

Figure 4. Confocal images and immunofluorescence data showing p-AMPK expression in the spinaldorsal horn. The quantification of p-AMPK immunofluorescence is presented as the mean fluorescence intensity and the number of positively stained cells $\left(\mathrm{n}=6 ;{ }^{* *} \mathrm{P}<0.01 \mathrm{vs}\right.$. sham $+\mathrm{NS}$;

${ }^{\# \#} \mathrm{P}<0.01 \mathrm{vs}$. CCI + NS; scale bar, $100 \mu \mathrm{m}$ ). Tissues were collected on day 10 after CCI. (p)-AMPK, phosphorylated adenosine monophosphate-activated protein kinase; NS, normal saline; CCI, chronic constriction injury. 


\section{A}
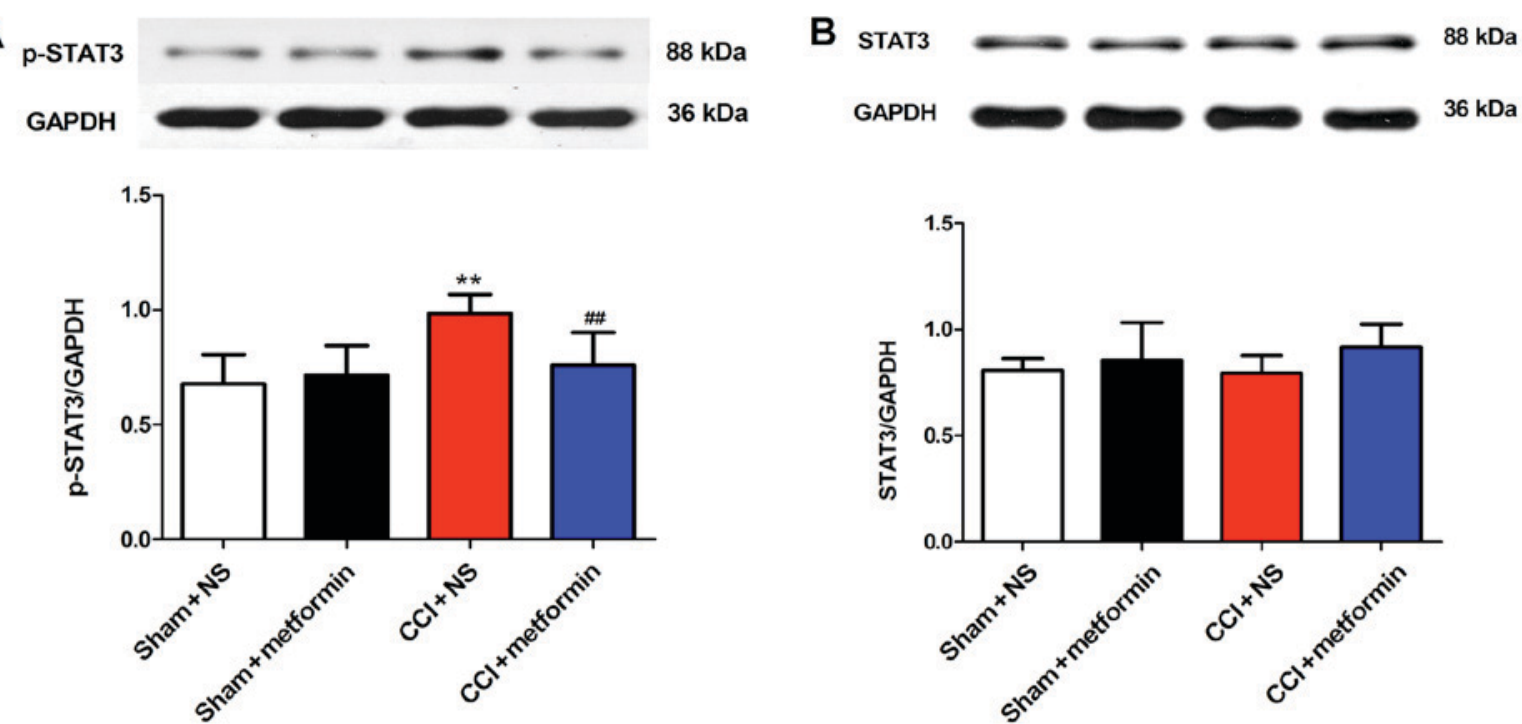

Figure 5. Metformin inhibited the activation of STAT3 in the spinal cords of CCI rats. (A) Metformin decreased the level of phosphorylated STAT3 in the spinal cord after CCI. ( $n=4 ;{ }^{* *} \mathrm{P}<0.01$ vs. sham $+\mathrm{NS} ;{ }^{\# \#} \mathrm{P}<0.01$ vs. CCI + NS) (B) There was no significant difference in the expression of STAT3 among the four groups ( $\mathrm{n}=4$; P>0.05). STAT3, signal transducer and activator of transcription 3; NS, normal saline; CCI, chronic constriction injury.

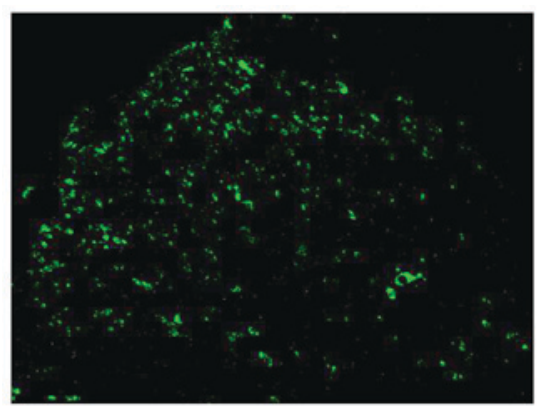

Sham + NS

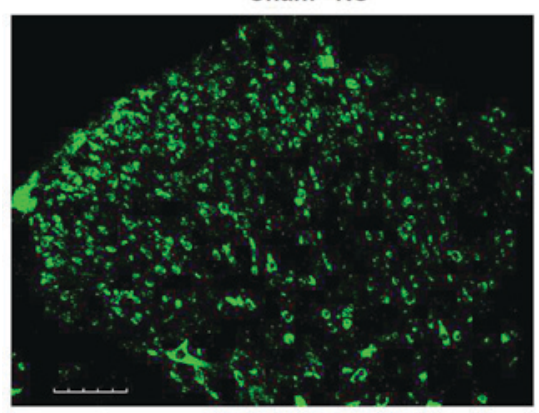

$\mathrm{CCl}+\mathrm{NS}$

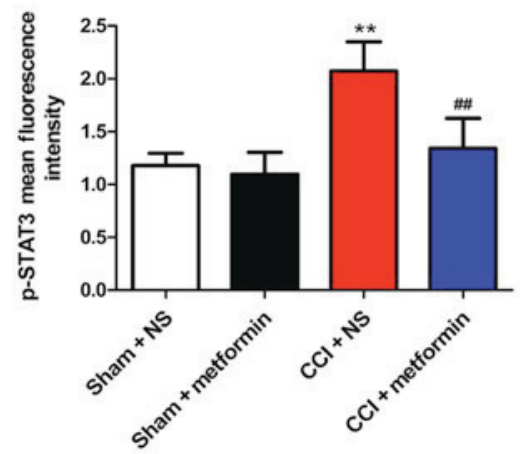

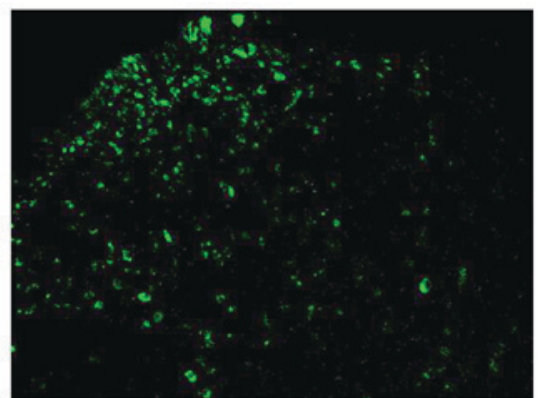

Sham + metformin

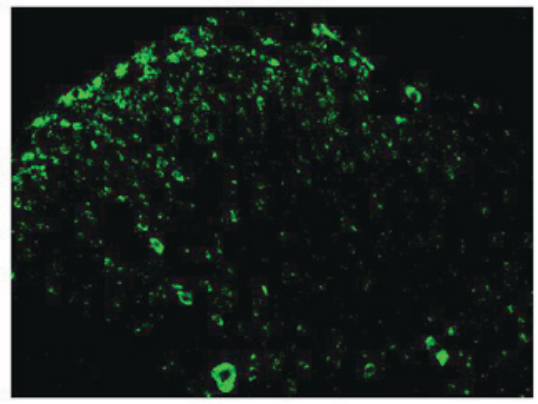

$\mathrm{CCl}+$ metformin

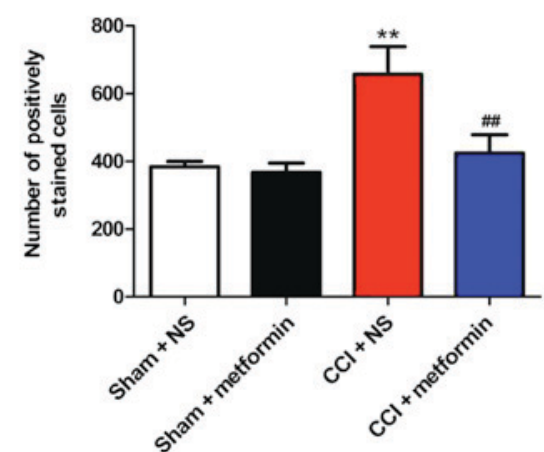

Figure 6. Confocal images and immunofluorescence analysis data showing p-STAT3 expression in the spinal dorsal horn. The quantification of p-STAT3 immunofluorescence is presented as the mean fluorescence intensity and the number of positively stained cells $\left(\mathrm{n}=6\right.$; ${ }^{* *} \mathrm{P}<0.01 \mathrm{vs}$. sham $+\mathrm{NS}$; ${ }^{\# \#} \mathrm{P}<0.01$ vs. CCI + NS; scale bar, $100 \mu \mathrm{m}$ ). Tissues were collected on day 10 after CCI. p-STAT3, phosphorylated signal transducer and activator of transcription 3; NS, normal saline; CCI, chronic constriction injury. 


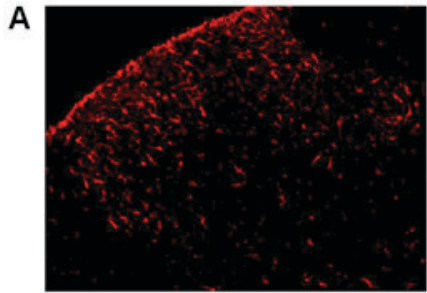

Sham + NS

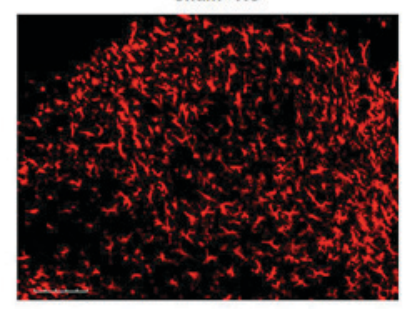

$\mathrm{CCl}+\mathrm{NS}$
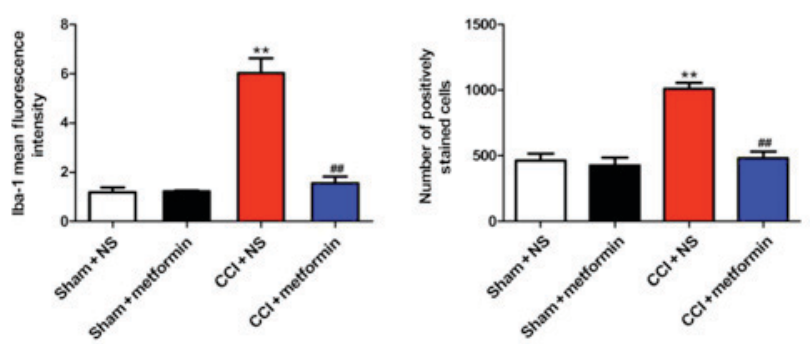

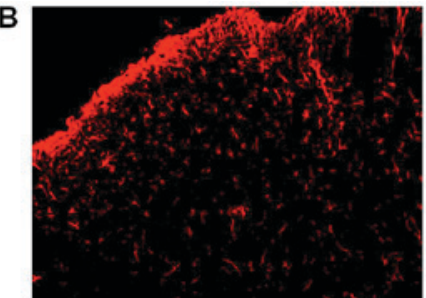

Sham+NS

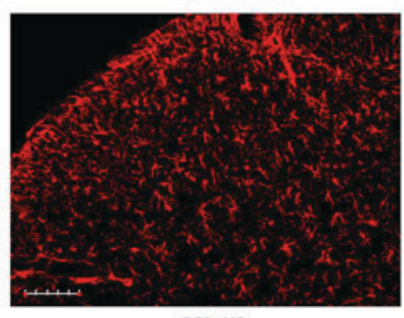

$\mathrm{CCl}+\mathrm{NS}$

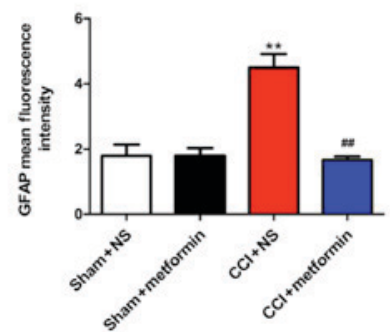

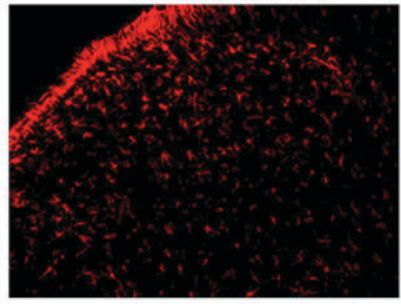

Sham+metformin

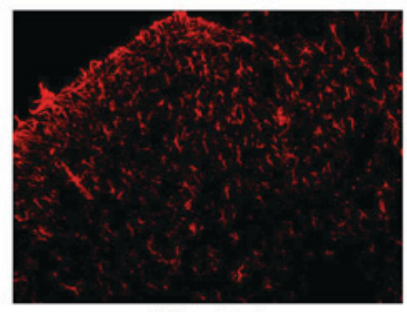

$\mathrm{CCl}+$ metformin

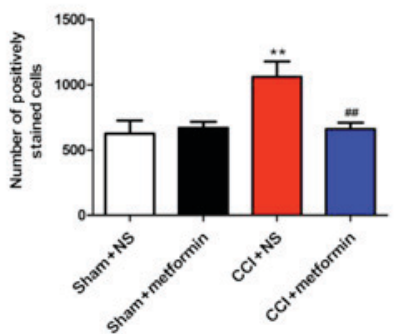

Figure 7. Confocal images and immunofluorescence staining for Iba-1 and GFAP expression in rat spinal dorsal horns. (A) The quantification of Iba-1 immunofluorescence staining is presented as the mean fluorescence intensity and the number of positively stained cells $\left(\mathrm{n}=6\right.$; ${ }^{* *} \mathrm{P}<0.01 \mathrm{vs}$. sham $+\mathrm{NS}$; ${ }^{\# \#} \mathrm{P}<0.01$ vs. CCI + NS; scale bar, $100 \mu \mathrm{m}$ ). (B) The quantification of GFAP immunofluorescence staining is presented as the mean fluorescence intensity and the number of positively stained cells $\left(\mathrm{n}=6 ;{ }^{* *} \mathrm{P}<0.01\right.$ vs. sham $+\mathrm{NS} ;{ }^{\#} \mathrm{P}<0.01$ vs. CCI $+\mathrm{NS}$; scale bar,100 $\left.\mu \mathrm{m}\right)$. Tissues were collected on day 10 after CCI. Iba-1, ionized calcium binding adaptor molecule 1; GFAP, glial fiber acidic protein; NS, normal saline; CCI, chronic constriction injury.

of astrocytes and microglia was effectively suppressed by metformin in CCI + metformin group $(\mathrm{P}<0.01)$ (Fig. 7).

\section{Discussion}

In the present study, the PWLs of the CCI rats dropped to a low level from day 3 to day 14. The expression of $\mathrm{p}$-AMPK was downregulated in the SDH in rats with chronic constriction injuries, while the expression of p-STAT3 was upregulated. On day 10 post-surgery, astrocytes and microglia were widely activated in these rats. However, administration of metformin $(200 \mathrm{mg} / \mathrm{kg})$ over 6 days effectively alleviated the thermal hyperalgesia and reversed the expression of $\mathrm{p}-\mathrm{AMPK}$ and p-STAT3, with the suppression of the activated microglia and astrocytes simultaneously.

This is the first study to explore the mechanism by which metformin inhibits NP in the SDH. The SDH is a complex relay station that transmits nociceptive information and occupies an important position in NP $(28,29)$. There are multiple pathways that transmit pain in the SDH, including the AMPK and STAT3 signaling pathways (11,16-18).

Metformin, an oral anti-diabetic drug, has been safely applied to treat type 2 diabetes for decades (30) and serves as an indirect agonist of AMPK. The increase of the cellular AMP/ATP ratio contributes to the activation of AMPK (9). Additionally, metformin promotes a shift from aerobic to anaerobic glycolysis by inhibiting complex I of the mitochondrial respiratory chain to increase the cellular AMP/ATP ratio $(12,13)$. In general, the chronic neuroinflammation in the nervous system contributes to the development of NP $(31,32)$. AMPK is a negative regulator of the mitogen-activated protein kinases and the mammalian target of rapamycin (mTOR) pathways, which are associated with the occurrence of chronic neuroinflammation (33-36). Further, AMPK can interfere with the synthesis of proteins by these pathways, and this has been linked to the development of chronic pain and the reduced expression of inflammatory factors. Thus, AMPK might be an ideal target for reducing or preventing hyperalgesia (9). In spinal nerve ligation (SNL) and spared nerve injury (SNI) models, metformin inhibited hyperalgesia and activated AMPK in vitro (5). Metformin also reduced diabetic NP and chemotherapy-induced NP in animal models $(37,38)$. In present study, we evidenced that metformin attenuates pain in CCI rats and suppresses the expression of p-AMPK, which may explain how metformin eases NP. The incidence of diabetic peripheral neuropathy (DPN) reportedly decreased by long-term oral administration of metformin, whereas the insulin treatment did not show this effect. In our study, there was insignificant difference of the blood glucose levels in rats with NP with or without the application of metformin, indicating that the analgesic effect of metformin is not associated with blood sugar levels, which is supported by the prior report (39).

STAT3 is an important component of the Janus kinase 2 (JAK2)-STAT3 signaling pathway. The JAK2-STAT3 signal pathway is widely involved in cell proliferation, differentiation, immune regulation and information transfer, which is 
involved in the formation of central sensitization in the spinal cord following peripheral nerve injury (40). Subsequent to the peripheral nerve damage, the inflammatory factor IL-6 is released and combines with the soluble IL- 6 receptor (sIL-6R) in the SDH, thus activating gpl30 on the cell surface and the JAK2-STAT3 signal transduction pathway, which is followed by the activation and proliferation of glial cells as well as the production and release of inflammatory cytokines $(40,41)$. These events result in a series of immune inflammatory reactions that enhance neuronal excitability and lead to NP. Recently, metformin is evidenced to repress the progression of cancers by the inhibition of the STAT3 signaling pathway $(14,15)$. There was a paucity of data regarding the effect of metformin on the STAT3 signaling pathway, with the underlying mechanisms remains elusive. AMPK is considered as a mediator between metformin and the STAT3 pathway $(42,43)$. Moreover, the activation of the STAT3 pathway depends on the activation of mTOR (44). Additionally, the activation of AMPK inhibited inflammation and suppressed the mTOR signaling pathway in a pain model $(5,11)$. In our experiments, mTOR may be the link between AMPK and the STAT3 pathway, and this may be the mechanism by which metformin inhibits STAT3 activation. We have therefore provided the first evidence showing that metformin decreases the activation of STAT3 in the SDH, which plays an important role in reducing NP.

The central nervous system consists of two types of cells-glia and neurons, the latter of which has been a heightened interest in the field of NP. Recently, emerging evidence focuses on the ion channels and neurotransmitter receptors on the membranes of glial cells. Glial cells can produce and release cytokines, which promote the development of NP $(20,21)$. Glia in the spinal cord are mainly composed of astrocytes, microglia and oligodendrocytes, with astrocytes being the most ones. Spinal astrocytes and microglia have small cell bodies with branched processes and are quiescent cells that monitor the local environment in the central nervous system under the normal conditions. In the event of an injury, spinal astrocytes and microglial are activated $(45,46)$, and synthesize and release the pro-inflammatory cytokines, which promote the neuroinflammation and NP. In our experiment, the glial cells and astrocytes were activated after CCI, which were effectively suppressed by metformin. The findings imply the involvement of glial cells and astrocytes in the pathogenesis of NP and the regulation of metformin for NP. In addition, STAT3 pathway was implicated in modulation of spinal glial cell proliferation and NP maintenance in rats $(17,23,40)$. In addition, AMPK is also involved in the functional regulation of astrocytes and microglia $(11,22,47)$. Hence, metformin activated AMPK and inhibited the activation of the STAT3 signaling pathway, both of which might further affect the functionality of astrocytes and microglia.

Previous studies have shown that the activation of microglia plays a role in the early stage of NP, while astrocyte activation takes effect in the maintenance phase of NP (48). We determined the distribution of astrocytes and microglia in the lumbar SDH on day 10 after sciatic nerve ligation, the time point of which is considered as a stable stage for CCI-induced NP. The L4-L6 spinal cord is the reference point for sciatic nerve lesion. Iba-1 and GFAP immunofluorescence staining results suggested that microglia and astrocytes were activated in the L4-L6 SDH. It indicated that the activation of microglia did not decrease and astrocytes have already been activated on day 10 after surgery. Our results confirm the previous claim that the activation of microglia and astrocytes may be an important step during the initiation and maintenance of NP after a peripheral nerve injury $(20,21,45)$. Our data show that metformin effectively inhibited the activation of spinal microglia and astrocytes, which might be an important mechanism by which metformin relieves NP.

In conclusion, intraperitoneal injections of metformin activated AMPK and inhibited the activation of the STAT3 signaling pathway in the SDH of CCI rats. Metformin could inhibit the activation of astrocytes and microglia, which might further inhibit the release of inflammatory cytokines, such as interleukin (IL-6, IL-1 $\beta$ ) and tumor necrosis factor $\alpha$ (TNF- $\alpha$ ), to alleviate NP. However, the limitation of our present study is that the colocalization of AMPK or STAT3 with glial cells was not presented. We will further study their relationship in the following studies.

The data in these experiments show that metformin is an effective and safe drug for NP. Given its affordability and easy accessibility, it is worthwhile to explore its clinical application in NP. Following further explorations of the characteristics of this drug and the mechanisms underlying NP, we believe that it is reasonable that this drug can be served as a potential therapeutic agent for chronic pain.

\section{Acknowledgements}

The present study was supported by grants from the National Natural Science Foundation of China (grant no. 81200861).

\section{References}

1. Jensen TS, Baron R, Haanpää M, Kalso E, Loeser JD, Rice AS and Treede RD: A new definition of neuropathic pain. Pain 152: 2204-2205, 2011.

2. Finnerup NB, Sindrup SH and Jensen TS: The evidence for pharmacological treatment of neuropathic pain. Pain 150: 573-581, 2010.

3. De Vry J,Kuhl E,Franken-KunkelP and Eckel G: Pharmacological characterization of the chronic constriction injury model of neuropathic pain. Eur J Pharmacol 491: 137-148, 2004.

4. Austin PJ, Wu A and Moalem-Taylor G: Chronic constriction of the sciatic nerve and pain hypersensitivity testing in rats. $\mathrm{J}$ Vis Exp: 13: 3393, 2012.

5. Melemedjian OK, Asiedu MN, Tillu DV, Sanoja R, Yan J, Lark A, Khoutorsky A, Johnson J, Peebles KA, Lepow T, et al: Targeting adenosine monophosphate-activated protein kinase (AMPK) in preclinical models reveals a potential mechanism for the treatment of neuropathic pain. Mol Pain 7: 70, 2011.

6. Taylor A, Westveld AH, Szkudlinska M, Guruguri P, Annabi E, Patwardhan A, Price TJ and Yassine HN: The use of metformin is associated with decreased lumbar radiculopathy pain. J Pain Res 6: 755-763, 2013.

7. Kim J, Yang G, Kim Y, Kim J and Ha J: AMPK activators: Mechanisms of action and physiological activities. Exp Mol Med 48: e224, 2016.

8. Dasgupta B and Chhipa RR: Evolving Lessons on the complex role of AMPK in normal physiology and cancer. Trends Pharmacol Sci 37: 192-206, 2016.

9. Price TJ and Dussor G: AMPK: An emerging target for modification of injury-induced pain plasticity. Neurosci Lett 557: 9-18, 2013.

10. Bullón P, Alcocer-Gómez E, Carrión AM, Marín-Aguilar F, Garrido-Maraver J, Román-Malo L, Ruiz-Cabello J, Culic O, Ryffel B, Apetoh L, et al: AMPK phosphorylation modulates pain by activation of NLRP3 inflammasome. Antioxid Redox Signal 24: 157-170, 2016. 
11. Song $\mathrm{H}$, Han Y, Pan C, Deng X, Dai W, Hu L, Jiang C, Yang Y, Cheng Z, Li F, et al: Activation of adenosine monophosphate-activated protein kinase suppresses neuroinflammation and ameliorates bone cancer pain. Anesthesiology 123: 1170-1185, 2015.

12. Hawley SA, Ross FA, Chevtzoff C, Green KA, Evans A, Fogarty S, Towler MC, Brown LJ, Ogunbayo OA, Evans AM and Hardie DG: Use of cells expressing gamma subunit variants to identify diverse mechanisms of AMPK activation. Cell Metab 11: 554-565, 2010.

13. Krishan S, Richardson DR and Sahni S: Adenosine monophosphate-activated kinase and its key role in catabolism: structure, regulation, biological activity and pharmacological activation. Mol Pharmacol 87: 363-377, 2015.

14. Liu Q, Yuan W, Tong D, Liu G, Lan W, Zhang D, Xiao H, Zhang Y, Huang Z, Yang J, et al: Metformin represses bladder cancer progression by inhibiting stem cell repopulation via COX2/PGE2/STAT3 axis. Oncotarget 7: 28235, 2016.

15. Park J, Kim WG, Zhao L, Enomoto K, Willingham $M$ and Cheng SY: Metformin blocks progression of obesity-activated thyroid cancer in a mouse model. Oncotarget 7: 34832, 2016.

16. Liu S, Li Q, Zhang MT, Mao-Ying QL, Hu LY, Wu GC, Mi WL and Wang YQ: Curcumin ameliorates neuropathic pain by down-regulating spinal IL-1 $\beta$ via suppressing astroglial NALP1 inflammasome and JAK2-STAT3 signalling. Sci Rep 6: 28956, 2016.

17. Tsuda M,Kohro Y, Yano T, Tsujikawa T,Kitano J, Tozaki-Saitoh H, Koyanagi S, Ohdo S, Ji RR, Salter MW and Inoue K: JAK-STAT3 pathway regulates spinal astrocyte proliferation and neuropathic pain maintenance in rats. Brain 134: 1127-1139, 2011.

18. Wang ZF, Li Q, Liu SB, Mi WL, Hu S, Zhao J, Tian Y, Mao-Ying QL, Jiang JW, Ma HJ, et al: Aspirin-triggered Lipoxin A4 attenuates mechanical allodynia in association with inhibiting spinal JAK2/STAT3 signaling in neuropathic pain in rats. Neuroscience 273: 65-78, 2014.

19. Park KW, Lin CY and Lee YS: Expression of suppressor of cytokine signaling-3 (SOCS3) and its role in neuronal death after complete spinal cord injury. Exp Neurol 261: 65-75, 2014.

20. Alfonso Romero-Sandoval E and Sweitzer S: Nonneuronal central mechanisms of pain: Glia and immune response. Prog Mol Biol Transl Sci 131:325-358, 2015.

21. Walters ET: Neuroinflammatory contributions to pain after SCI Roles for central glial mechanisms and nociceptor-mediated host defense. Exp Neurol 258: 48-61, 2014.

22. Gui Y, Li A, Chen F, Zhou H, Tang Y, Chen L, Chen S and Duan S: Involvement of AMPK/SIRT1 pathway in anti-allodynic effect of troxerutin in CCI-induced neuropathic pain. Eur J Pharmacol 769: 234-241, 2015.

23. Molet J, Mauborgne A, Diallo M, Armand V, Geny D, Villanueva L, Boucher Y and Pohl M: Microglial Janus kinase/signal transduction and activator of transcription 3 pathway activity directly impacts astrocyte and spinal neuron characteristics. J Neurochem 136: 133-147, 2016.

24. Gerard E, Spengler RN, Bonoiu AC, Mahajan SD, Davidson BA, Ding H, Kumar R, Prasad PN, Knight PR and Ignatowski TA Chronic constriction injury-induced nociception is relieved by nanomedicine-mediated decrease of rat hippocampal tumor necrosis factor. Pain 156: 1320-1333, 2015.

25. Hargreaves K, Dubner R, Brown F, Flores C and Joris J: A new and sensitive method for measuring thermal nociception in cutaneous hyperalgesia. Pain 32: 77-88, 1988.

26. Zhang T, Sun K, Shen W, Qi L, Yin W and Wang LW: SOCS1 regulates neuropathic pain by inhibiting neuronal sensitization and glial activation in mouse spinal cord. Brain Res Bull 124 231-237, 2016

27. Cao $\mathrm{H}$ and Zhang YQ: Spinal glial activation contributes to pathological pain states. Neurosci Biobehav Rev 32: 972-983, 2008.

28. Benarroch EE: Dorsal horn circuitry Complexity and implications for mechanisms of neuropathic pain. Neurology 86 1060-1069, 2016

29. West SJ, Bannister K, Dickenson AH and Bennett DL: Circuitry and plasticity of the dorsal horn-Toward a better understanding of neuropathic pain. Neuroscience 300: 254-275, 2015.

30. Martin M and Marais R: Metformin: A diabetes drug for cancer, oral cancer drug for diabetics? J Clin Oncol 30: 2698-2700, 2012.
31. Wan W, Cao L, Khanabdali R, Kalionis B, Tai X and Xia S: The emerging role of HMGB1 in neuropathic pain: A Potential therapeutic target for neuroinflammation. J Immunol Res 2016: 6430423, 2016

32. Zhou YQ, Liu Z, Liu ZH, Chen SP, Li M, Shahveranov A, Ye DW and Tian YK: Interleukin-6: An emerging regulator of pathological pain. J Neuroinflammation 13: 141, 2016.

33. Edelmayer RM, Brederson JD, Jarvis MF and Bitner RS: Biochemical and pharmacological assessment of MAP-kinase signaling along pain pathways in experimental rodent models: A potential tool for the discovery of novel antinociceptive therapeutics. Biochem Pharmacol 87: 390-398, 2014.

34. Lin X, Wang M, Zhang J and Xu R: p38 MAPK: A potential target of chronic pain. Curr Med Chem 21: 4405-4418, 2014.

35. Lisi L, Aceto P, Navarra P and Dello Russo C: MTOR kinase: A possible pharmacological target in the management of chronic pain. Biomed Res Int 2015: 394257, 2015.

36. Sanna MD, Stark H, Lucarini L, Ghelardini C, Masini E and Galeotti N: Histamine H4 receptor activation alleviates neuropathic pain through differential regulation of ERK, JNK and P38 MAPK phosphorylation 156: 2492-2504, 2015.

37. Ma J, Yu H, Liu J, Chen Y, Wang Q and Xiang L: Metformin attenuates hyperalgesia and allodynia in rats with painful diabetic neuropathy induced by streptozotocin. Eur J Pharmacol 764: 599-606, 2015.

38. Mao-Ying QL, Kavelaars A, Krukowski K, Huo XJ, Zhou W, Price TJ, Cleeland C and Heijnen CJ: The anti-diabetic drug metformin protects against chemotherapy-induced peripheral neuropathy in a mouse model. PLoS One 9: e100701, 2014.

39. Pop-Busui R, Lu J, Lopes N and Jones TL; BARI 2D Investigators: Prevalence of diabetic peripheral neuropathy (DPN) and relation to glycemic control strategies at baseline in the BARI 2D cohort. J Peripher Nerv Syst 14: 1-13, 2009.

40. Dominguez E, Rivat C, Pommier B, Mauborgne A and Pohl M: JAK/STAT3 pathway is activated in spinal cord microglia after peripheral nerve injury and contributes to neuropathic pain development in rat. J Neurochem 107: 50-60, 2008.

41. Dubový P, Klusáková I, Svízenská I and Brázda V: Satellite glial cells express IL-6 and corresponding signal-transducing receptors in the dorsal root ganglia of rat neuropathic pain model. Neuron Glia Biol 6: 73-83, 2010.

42. Yue W, Zheng X, Lin Y, Yang CS, Xu Q, Carpizo D, Huang H, DiPaola RS and Tan XL: Metformin combined with aspirin significantly inhibit pancreatic cancer cell growth in vitro and in vivo by suppressing anti-apoptotic proteins Mcl-1 and Bcl-2. Oncotarget 6: 21208-21224, 2015.

43. Hattori Y, Hattori K and Hayashi T: Pleiotropic benefits of metformin: Macrophage targeting its anti-inflammatory mechanisms. Diabetes 64: 1907-1909, 2015.

44. Yokogami K, Wakisaka S, Avruch J and Reeves SA: Serine phosphorylation and maximal activation of STAT3 during CNTF signaling is mediated by the rapamycin target mTOR. Curr Biol 10: 47-50, 2000.

45. Tsuda M: Microglia in the spinal cord and neuropathic pain. J Diabetes Investig 7: 17-26, 2016.

46. Nimmerjahn A, Kirchhoff $F$ and Helmchen F: Resting microglial cells are highly dynamic surveillants of brain parenchyma in vivo. Science 308: 1314-1318, 2005.

47. Maixner DW, Yan X, Gao M, Yadav R and Weng HR: Adenosine Monophosphate-activated protein kinase regulates interleukin-1 $\beta$ expression and glial glutamate transporter function in rodents with neuropathic pain. Anesthesiology 122: $1401-1413,2015$.

48. Hald A, Nedergaard S, Hansen RR, Ding M and Heegaard AM: Differential activation of spinal cord glial cells in murine models of neuropathic and cancer pain. Eur J Pain 13: 138-145, 2009.

This work is licensed under a Creative Commons Attribution-NonCommercial-NoDerivatives 4.0 International (CC BY-NC-ND 4.0) License. 